\title{
Epidemiological typing of Streptococcus pyogenes by pyrolysis mass spectrometry
}

\author{
J. T. MAGEE, J. M. HINDMARCH*, I. A. BURNETT* and A. PEASE* \\ Department of Microbiology, Children's Hospital, Sheffield S10 2TH and *Department of Bacteriology, Royal \\ Hallamshire Hospital, Sheffield S102JF
}

\begin{abstract}
Summary. Strains of Streptococcus pyogenes from an outbreak of infection on a burns unit(15), a collection of routine isolates from another hospital(12) and isolates from a national survey of throat infections in children in the community(4) were examined blind by pyrolysis-mass spectrometry (Py-MS). The outbreak strains (M22 T12) previously found to give identical typing results in conventional tests, formed a closely similar cluster and were distinct from other hospital and community strains. One hospital and one community strain were loosely associated with this cluster. Another cluster comprised six antibiotic-susceptible strains and two community strains. Six strains did not fall within the clusters; four were antibiotic-resistant strains isolated in hospital, one an antibiotic-resistant strain isolated in the community, and one a susceptible hospital strain. Results show that Py-MS is a potentially valuable method for rapid comparison of strains in studies of infection.
\end{abstract}

\section{Introduction}

Pyrolysis-mass spectrometry (Py-MS) is a rapid technique in which a sample of organic material is heated to $>400^{\circ} \mathrm{C}$ in a vacuum and the volatile products formed by thermal degradation - the pyrolysate - are separated and quantitated by mass spectrometry. The quantitative and qualitative composition of the pyrolysate reflect the composition of the sample and the conditions of pyrolysis. For samples of complex organic material the pyrolysis-mass spectrum may be regarded as providing 'fingerprint' data. When applied to microorganisms, Py-MS provides data which reflect overall cell composition. The technique has been used in classification, identification and typing of micro-organisms (Gutteridge and Norris, 1979; Drucker, 1981; Gutteridge et al., 1985; Shute et al., 1985). Rapid typing methods are important in studying outbreaks of infection, and a recent outbreak of infection with Streptococcus pyogenes type T12 M22 in a burns unit (Burnett and Norman, in press) provided the opportunity to assess the value of Py-MS as a typing technique.

\section{Materials and methods}

\section{Strains of S. pyogenes}

The 31 strains examined comprised 15 isolates from

Received 16 June 1989; accepted 22 June 1989. the outbreak at the burns unit of the Northern General Hospital, Sheffield, 12 from a collection of strains isolated from routine specimens at the Royal Hallamshire Hospital (RHH), and four isolates from a national survey of community-acquired throat infections in children. All were Lancefield group A $\beta$-haemolytic streptococci. The outbreak strains had previously been typed as T12 M22 (Burnett and Norman, in press). Four of the RHH strains and one of the survey strains showed resistance to tetracycline, erythromycin or both, in vitro. Cultures were stored at $-70^{\circ} \mathrm{C}$ in $10 \%$ glycerol broth.

\section{Preparation of cultures for pyrolysis}

Test strains were grown aerobically at $37^{\circ} \mathrm{C}$ on blood agar (Columbia Agar Base, Oxoid, with horse blood, CC Laboratories, $5 \%$ ) for $24 \mathrm{~h}$. Thirty-five cultures were examined, comprising one of each of the 31 isolates, and duplicate cultures of two isolates from the outbreak set, one from the RHH set, and one from the survey. These 35 cultures were blind-coded independently and the code was not broken until mathematical analysis of the results was complete.

\section{Pyrolysis-mass spectrometry}

Py-MS was performed on a PYMS 200X pyrolysismass spectrometer (Pi-Rho Technology, Heathfield, Sussex) as previously described (Aries et al., 1986; Magee et al., 1989). Growth from 24-h cultures on blood agar was smeared on iron-nickel alloy pyrolysis foils and heated rapidly in a vacuum to $530^{\circ} \mathrm{C}$ by the Curie point method. The volatile low mol. wt pyrolysis products were 
ionised by low energy electron impact and separated according to mass-charge ratio in the quadrupole mass spectrometer to yield a quantitative mass spectrum. Quadruplicate analyses were performed on each culture. Mathematical analysis was as described previously (Duerden et al., 1989). Spectra were normalised to correct for variation in sample size on the basis of total ion count for masses $51-63,65-72,74,75,77-86,89-96,98-100$, $103,104,106,107,116$ and 127 . Inter-strain discriminant analysis of normalised ion counts for these 50 masses yielded 17 canonical discriminant functions (CDFs). These may be regarded as statistically corrected variables describing the significant inter-strain spectrum differences. The sum of the squares of the differences between these CDF's for any two strains corresponds to the $\chi^{2}$ (16df) probability that the spectra originated from the same strain. Cluster analysis of the CDF data, by UPGMA and Euclidian distance ${ }^{2}$ yielded the dendrogram (fig. 1). The co-phenetic correlation was 0.915 . The ordination diagram (fig. 2) represents $64 \%$ of the interstrain variation as a scatter diagram on the first three CDFs.

\section{Results}

The dendrogram (fig. 1) shows a tightly homogeneous cluster $\left(\chi^{2} c .323, \mathrm{p}<0.001\right)$ comprising mean spectra for 16 cultures with three further cultures which were less similar but clearly associated; all were designated cluster A. A second cluster (B) comprised mean spectra for 10 cultures. Mean spectra for a further six cultures were dissimilar to one another and to clusters A and B. The spectra of all 15 isolates from the outbreak came within cluster A, including those for both cultures of the two isolates submitted in duplicate. The three strains that lay outwith the main body of cluster A comprised one RHH strain, one survey strain, and the duplicate culture of one of the outbreak strains. Cluster B comprised three community strains, including both duplicate cultures of one of these, and five RHH strains, again with both duplicate cultures of one of these strains. The six strains that did not fit into the cluster pattern comprised all four antibiotic-resistant strains from the RHH set, the single antibiotic resistant strain from the community survey, and one susceptible strain from the RHH set. All five antibiotic-resistant strains showed major differences in composition from the strains of the two clusters and from each other.

The ordination diagram (fig. 2) showed a roughly spherical group corresponding to cluster $\mathrm{A}$ and a more dispersed group corresponding to cluster B with the distinct antibiotic-sensitive strain and two antibiotic-resistant strains as outwith the groups. The three remaining antibiotic-resistant strains were omitted from this diagram because they were so far outwith the main clusters that a fourfold reduction in axis scales would have been necessary for their inclusion.

\section{Discussion}

The results show the potential of Py-MS as an epidemiological typing system. The outbreak strains were closely similar, and were clearly distinguished from the other strains. Other studies have shown that Py-MS can be used in inter-strain discrimination of a wide range of micro-organisms, including Listeria monocytogenes (Eshuis et al., 1977), Neisseria gonorrhoeae (Borst et al., 1978), Klebsiella aerogenes (Meuzelaar et al., 1982), Bacillus thuringiensis (Gutteridge et al., 1985), B. cereus (Shute et al., 1986) and Candida albicans (Magee et al., 1988) but none of these was studied in the context of hospital infection.

Typing by Py-MS differs from conventional typing in several aspects (see table). In Py-MS typing many continuously variable characters containing information on many aspects of the cell are processed statistically to yield probabilities that spectra are derived from the same strain. This avoids the 'type' concept of infra-species groups of strains that give identical results because of similarity rather than because of a common origin. Such type groups may exist in Py-MS in terms of infraspecies groups that yield similar spectra (see below), but the large number of characters examined make unlikely spectra that are fortuitously identical. Therefore, there is little evidence that the type concept, as accepted in conventional techniques, is relevant to Py-MS.

In hospital infection investigations typing is performed to determine whether strains are 'indistinguishable', i.e., not significantly dissimilar in typing characteristics. As conventional typing deals with presence-or-absence attributes, mathematical processing is not necessary and results are represented as a simple code. By contrast, the data from Py-MS are complex and require analysis by multivariate statistical techniques; the results, however, are objective statistical probabilities. No strains are 'untypable' in this approach.

In conventional methods the number of cell components investigated and the number of possible states of the characters examined is small. It is likely that chance coincidence of typing markers will occur, making otherwise dissimilar strains indistinguishable. Py-MS data comprises many continuous (quantitative) variables - the ion intensities for each mass resolved-that probably reflect the organic composition of most major cell compo- 


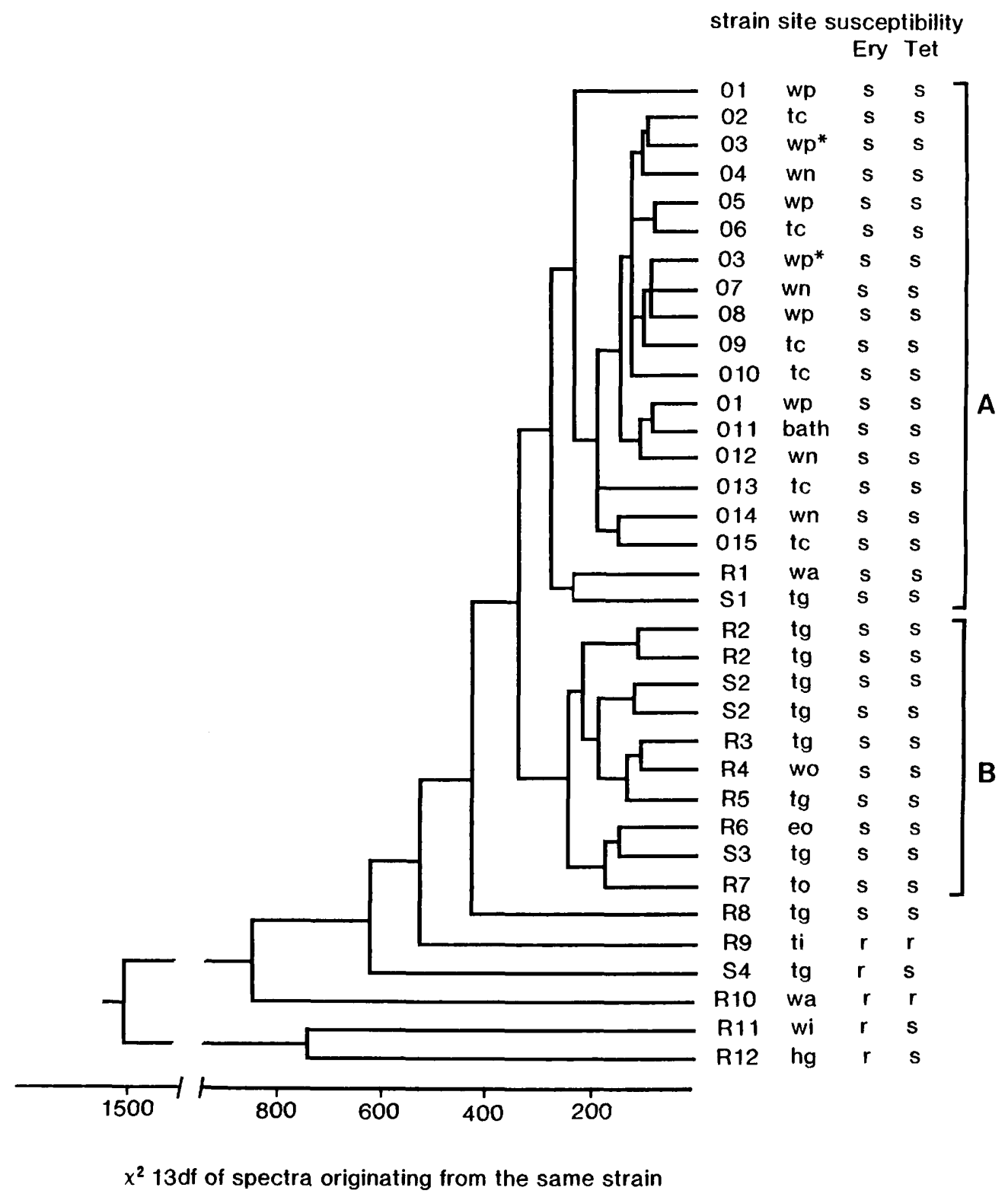

Fig. 1. A dendrogram representing inter-strain similarities in Py-MS. Site abbreviations for the outbreak strains (OI-OI5) are: wp-wound site, patient; wn-lesion, nurse; tc - throat of a member of the index case's family; bath-an isolate from a bath in the unit. *The index case strain. Abbreviations for the hospital (R1-R12) and community survey (S1-S4) strains are: wa, wi, wo-wound site casualty, in- or out-patient; $\mathrm{tg}$, ti, to - throat of general practice, in- or out-patient; eo-ear, out patient; hg-high vaginal, general practice.

nents, and may be less susceptible to false assignment of strains as indistinguishable.

Although Py-MS requires large initial capital expenditure for apparatus, running costs are low, contrasting with conventional methods that require minimal expenditure on apparatus but need a wide range of labile reagents and considerable effort in the development and maintainance of each method to cover a wide bacterial spectrum.

Other recently developed typing methods, e.g., SDS-PAGE (Jackman, 1985), immune blotting (Burnie and Matthews, 1987) and restriction enzyme analysis of nucleic acids (Bjorvain and Kristiansen, 1985), show many characteristics 


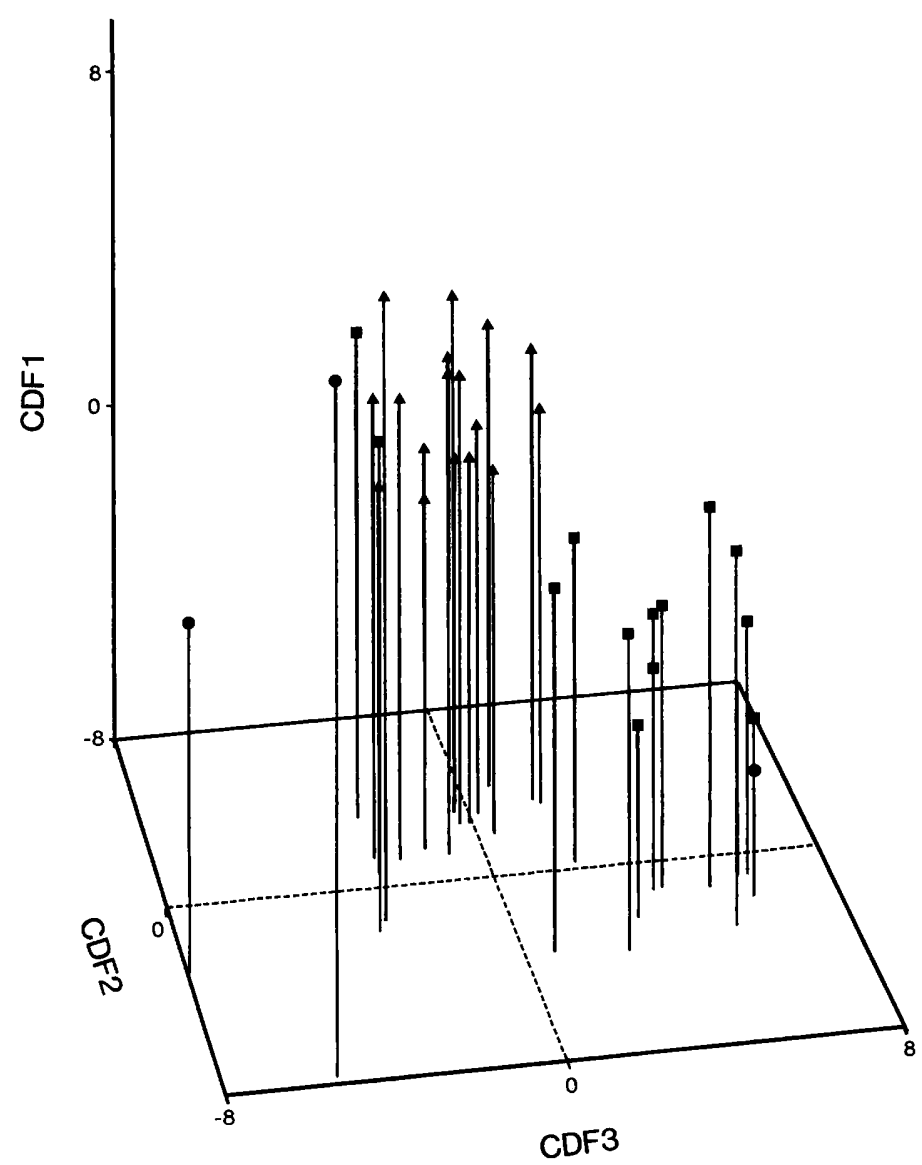

Fig. 2. A pseudo-three dimensional ordination diagram showing the scatter of mean strain spectrum points on the first three canonical discriminant function axes (CDF 1-3). These axes account for $64 \%$ of the statistically significant inter-strain discrimination. Outbreak strains, $\mathbf{\Delta}$; non-outbreak strains, $\mathbf{\square}$; non-cluster strains,

Table. A comparison of Py-MS and conventional typing methods

\begin{tabular}{lcc}
\hline & \multicolumn{2}{c}{ Typing technique } \\
\cline { 2 - 3 } Features of methods & Conventional & Py-MS \\
\hline Number of characters examined & few & many \\
Range of cell constituents examined & few/one & all/most \\
Untypable strains & yes & no \\
Species specific methodology & yes & no \\
Many reagents with limited shelf life & yes & no \\
Specialised apparatus & no & yes \\
Computational processing & no & essential \\
Probability of strains being identical given & no & yes \\
Clonal stability of markers & yes & unknown \\
Stability of method & yes & unknown \\
Inter-laboratory reproducibility & yes & unknown \\
Existence of type groups & yes & unknown \\
Pathogenic significance of type & sometimes & unknown \\
& & \\
\hline
\end{tabular}


similar to those of Py-MS. The data yielded are multivariate and, as they are potentially quantitative, might be amenable to statistical analysis similar to that used in the present study. Indistinguishable strains of distinct origin are encountered comparatively infrequently and no strains are untypable. Also, these techniques are applicable, with minor modifications, to a broad range of pathogenic species.

Some important properties of Py-MS have yet to be defined. For example, long term stability of the apparatus and methodology, i.e., the comparability of spectra obtained for single strains in repeated analyses over a prolonged period at the detailed level examined in typing, has not been documented; nor has the stability of the Py-MS strain 'markers', i.e., the typing similarity of the spectra of single strains following prolonged passage in vitro or from fresh clinical specimens. In this study, Py-MS strain markers remained stable for the duration of the outbreak, isolates from the index case, patients, contacts, and environment showing close similarity. Clearly these isolates were separated from their hypothetical common origin by many growth cycles, both from fresh cultures and in later laboratory culture and storage. One strain (O 5) isolated a month after the onset of the outbreak remained closely similar to the index strain. In the context of typing inter-laboratory reproducibility of results remains completely unknown.

The applicability of the type concept in Py-MS is at present uncertain; there is no clear evidence for a consistent 'granularity' in the infra-species distribution on the basis of overall cell composition, i.e., the potential of micro-organisms to be divided

\section{REFERENCES}

Aries R E, Gutteridge C S, Ottley T W 1986 Evaluation of a low-cost, automated pyrolysis-mass spectrometer. Journal of Analytical and Applied Pyrolysis 9: 81-98.

Bjorvain B, Kristiansen B-E 1985 Molecular epidemiology of bacterial infections. Clinics in Laboratory Medicine 5 : 437455.

Borst J, Van Der Snee-Enkelaar A C, Meuzelaar H L C 1978 Typing of Neisseria gonorrhoeae by pyrolysis mass spectrometry. Antonie van Leeuwenhoek 44: 253.

Burnett I A, Norman P Streptococcus pyogenes: an outbreak on a burns unit. Journal of Hospital Infection (in press).

Burnie J P, Matthews R C 1987 Immunoblot analysis: a new method for fingerprinting hospital pathogens. Journal of Immunological Methods 100: 41-46.

Dillon H C, Derrick C W, Dillon M S 1974 M-antigens common to pyoderma and acute glomerulonephritis. Journal of Infectious Diseases 130: 257-267.

Drucker D B 1981 Microbiological applications of gas chromatography. Cambridge University Press, Cambridge. into infra-species groups on the basis of similarity of their pyrolysis-mass spectra. However, infraspecies Py-MS groups were found in Corynebacterium jeikeium (Hindmarch et al., in press) and, in this survey, in $S$. pyogenes as seen by the loose association of unrelated strains in cluster B. In conventional typing certain type groups are associated with particular pathological manifestations, e.g., the association of pyoderma and glomerulonephritis with M-type $49 S$. pyogenes infections (Dillon et al., 1974). The only evidence of association between pathogenicity and Py-MS properties is that of Shute et al. (1986), who found that strains of $B$. cereus producing vomiting, diarrhoea or no symptoms and signs were discriminated by Py-MS. In our study there was a significant association between antibiotic resistance and gross dissimilarity to the two major clusters of susceptible strains. Whether such associations will be exceptional or common remains unknown. Clearly, studies of larger scope on many pathogenic species are required to establish these relationships.

Investigation of Py-MS typing in point source hospital outbreaks of short duration avoids the theoretical problems of long term reproducibility outlined above. In this context Py-MS is a potentially useful typing tool, but much further work is required if this technique is to be used in the study of geographically dispersed infections of common origin.

We thank the Trent Regional Health Authority Research Committee for support of earlier work, which allowed the development of the practical and mathematical approaches used here, Perkin-Elmer Ltd for the loan of the PYMS 200X and Dr P. Norman for the collection of outbreak strains.
Duerden B I, Eley A, Goodwin L, Magee J T, Hindmarch J M, Bennett K W 1989 A comparison of Bacteroides ureolyticus isolates from different clinical sources. Journal of Medical Microbiology 29 : 63-73.

Eshuis W, Kistemaker P G, Meuzelaar H L C 1977 Some numerical aspects of reproducibility and specificity. In: Jones C E R, Cramers C A (eds) Analytical pyrolysis. Elsevier, Amsterdam, pp 151-166.

Gutteridge C S, Norris J R 1979 The application of pyrolysis techniques to the identification of micro-organisms. Journal of Applied Bacteriology 47: 5-43.

Gutteridge C S, Vallis L, MacFie H J H 1985 Numerical methods in the classification of micro-organisms by pyrolysis mass spectrometry. In : Goodfellow M, Jones D, Priest F G (eds) Computer assisted bacterial systematics. Academic Press, London, pp 369-401.

Hindmarch J M, Magee J T, Hadfield M, Duerden B I A pyrolysis mass spectrometry study of Corynebacterium spp. and Listeria monocytogenes. Journal of Medical Microbiology (in press).

Jackman H J H 1985 Bacterial taxonomy based on electropho- 
retic whole-cell protein patterns. In: Goodfellow $\mathrm{M}$, Minnikin D E (eds) Chemical methods in bacterial systematics. Academic Press, London, pp 115-129.

Magee J T, Hindmarch J M, Bennett K W, Duerden B I, Aries R E 1989 A pyrolysis mass spectrometry study of fusobacteria. Journal of Medical Microbiology 28: 227--236.

Magee J T, Hindmarch J M, Duerden B I, Mackenzie D W R 1988 Pyrolysis-mass spectrometry as a method for interstrain discrimination of Candida albicans. Journal of General Microbiology 134: 2841-2847.

Meuzelaar H L C, Haverkamp J, Hileman F D 1982 Pyrolysis mass spectrometry of recent and fossil biomaterials. Compendium and atlas. Elsevier, Amsterdam.

Shute L A, Berkeley R C W, Norris J R, Gutteridge C S 1985 Pyrolysis mass spectrometry in bacterial systematics. In: Goodfellow M, Minnikin D E (eds) Chemical methods in bacterial systematics. Academic Press, London, pp 95-114.

Shute L A, Gutteridge C S, Berkeley R C W 1986 Pyrolysis mass spectrometry: a discriminatory technique with potential for rapid characterization and identification of microorganisms. Abstracts, 'Microbe 86' XIV International Congress of Microbiology. 7-13 September 1986, Manchester. p 75. 